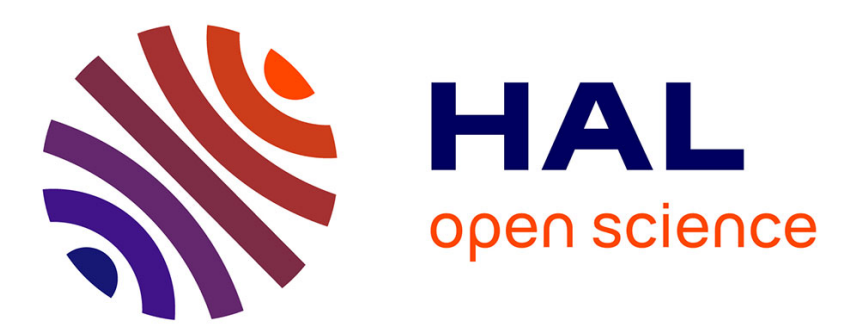

\title{
Experimental observation of the elliptically polarized fundamental vector soliton of isotropic Kerr media
}

\author{
M. Delque, T. Sylvestre, H. Maillotte, Cyril Cambournac, P. Kockaert, M.
} Haelterman

\section{- To cite this version:}

M. Delque, T. Sylvestre, H. Maillotte, Cyril Cambournac, P. Kockaert, et al.. Experimental observation of the elliptically polarized fundamental vector soliton of isotropic Kerr media. Optics Letters, 2005, 30, n² 24, pp.3383-3385. hal-00097385

\section{HAL Id: hal-00097385 \\ https://hal.science/hal-00097385}

Submitted on 26 Aug 2013

HAL is a multi-disciplinary open access archive for the deposit and dissemination of scientific research documents, whether they are published or not. The documents may come from teaching and research institutions in France or abroad, or from public or private research centers.
L'archive ouverte pluridisciplinaire HAL, est destinée au dépôt et à la diffusion de documents scientifiques de niveau recherche, publiés ou non, émanant des établissements d'enseignement et de recherche français ou étrangers, des laboratoires publics ou privés. 


\title{
Experimental observation of the elliptically polarized fundamental vector soliton of isotropic Kerr media
}

\author{
Michaël Delqué, Thibaut Sylvestre, and Hervé Maillotte \\ Département d'Optique P.M. Duffieux, Institut FEMTO-ST, Université de Franche-Comté, Centre National de la \\ Recherche Scientifique, Unité Mixte de Recherche 6174, F-25030 Besançon, France \\ Cyril Cambournac, Pascal Kockaert, and Marc Haelterman \\ Service d'Optique et d'Acoustique, Université Libre de Bruxelles, B-1050 Brussels, Belgium
}

Received May 25, 2005; revised manuscript received July 18, 2005; accepted August 10, 2005

We report the experimental observation of the elliptically polarized fundamental vector soliton of isotropic Kerr media and its unique polarization evolution. This was achieved in the spatial domain in a nonbirefringent $\mathrm{CS}_{2}$ planar waveguide. (C) 2005 Optical Society of America

OCIS codes: $190.5530,190.4420,190.3270$.

The vector nature of polarized light propagating in nonlinear dispersive or diffractive media ${ }^{1-3}$ has led to the discovery of a novel class of solitary-wave solution of the two coupled nonlinear Schrödinger (NLS) equations. ${ }^{4-8}$ As initially demonstrated theoretically in birefringent media by Christodoulides and Joseph and by Tratnik and Sipe, ${ }^{8}$ these solutions are stationary polarization bound states, thus leading to the concept of the vector soliton. A few years later, Haelterman and Sheppard ${ }^{9}$ demonstrated analytically and numerically the existence of such vector solitons in isotropic Kerr media, i.e., without linear birefringence. In addition to both the well-known linearly and circularly polarized NLS solitons, they found new solutions that consist of elliptically polarized fundamental vector solitons (EPVSs). In particular, they exhibit a nonuniform transverse ellipticity profile, and their global polarization state regularly rotates during propagation. This property is reminiscent of the self-induced nonlinear birefringence that is due to cross-phase modulation between the two circular polarization components of the soliton. Until now, to our knowledge no experimental demonstration of EPVSs in isotropic media had been made. In this Letter we report the experimental observation and characterization of a spatial EPVS generated in a Kerr liquid planar waveguide.

The experiment, schematically sketched in Fig. 1, is performed in a $3 \mathrm{~cm}$ long $\mathrm{CS}_{2}$ liquid planar slab waveguide. The step-index waveguide is made from a $15 \mu \mathrm{m}$ thick $\mathrm{CS}_{2}$ layer sandwiched between two SK5 glass plates, whose index difference is $\Delta n=0.04{ }^{10} \mathrm{~A}$ beat length of $L=1.7 \mathrm{~m}$ much longer than the waveguide length was measured, which ensures the quasiisotropic condition to generate and maintain the EPVS. As a pump laser source we use a compact passively $Q$-switched microchip Nd:YAG laser emitting $600 \mathrm{ps}$ Gaussian pulses at a repetition rate of $6.7 \mathrm{kHz}$ and at a wavelength of $532 \mathrm{~nm}$ (the mean power is 30 $\mathrm{mW})$. The high power stability $(<1 \% \mathrm{rms})$ as well as the smooth circular $\mathrm{TEM}_{00}$ beam profile of the laser allows one to generate a spatial soliton with excellent transverse stability and therefore to measure its polarization state accurately. The elliptic polarization state of the input beam was adjusted by means of a quarter-wave plate. A couple of cylindrical lenses $(\mathrm{L} x 1, \mathrm{~L} x 2)$ were inserted to adjust horizontal size $x$ of the beam (soliton width) without changing vertical size $y$. At the waveguide's output, a second quarterwave plate and a Wollaston biprism (W) were used to extract either the $\left(E_{x}, E_{y}\right)$ linearly polarized components or the $(U, V)$ circularly polarized components, depending on orientations on the wave plate and the prism. First, to get access to the global polarization state we directly observe the energy ratio between the linearly polarized components. Second, we measure the ellipticity factor across the transverse beam profile by means of the circularly polarized components, using the following definition: $q(x)=\left[|U(x)|^{2}\right.$ $\left.-|V(x)|^{2}\right] /\left[|U(x)|^{2}+|V(x)|^{2}\right]$.

The results of our experiment are detailed in Fig. 2. Figures 2(a)-2(c) illustrate the $E_{x}$ and $E_{y}$ profiles at the waveguide's input and output in linear and soliton regimes, respectively. Figures 2(d)-2(f) exhibit the $U$ and $V$ intensity profiles in the same conditions. Additionally, in Figs. 2(d)-2(f) we have plotted (dotted curves) measured ellipticity factor $q$. Let us recall that the values $q= \pm 1,0$ represent circular and linear polarization, respectively. The first quarter-wave plate is then tuned such that the input ellipticity factor is 0.29 , and it is of course constant all across the transverse beam profile, as shown by the dotted curve in Fig. 2(d). For this degree of ellipticity, one can see from Fig. 2(a) that the power of the $E_{y}$ polarization is much less than that of the $E_{x}$ polarization. Figures 2(b) and 2(e) show the same pro-

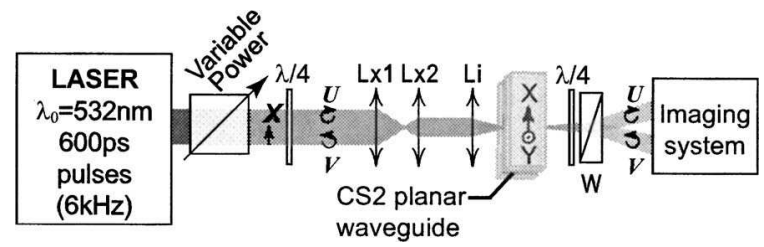

Fig. 1. Experimental setup. 


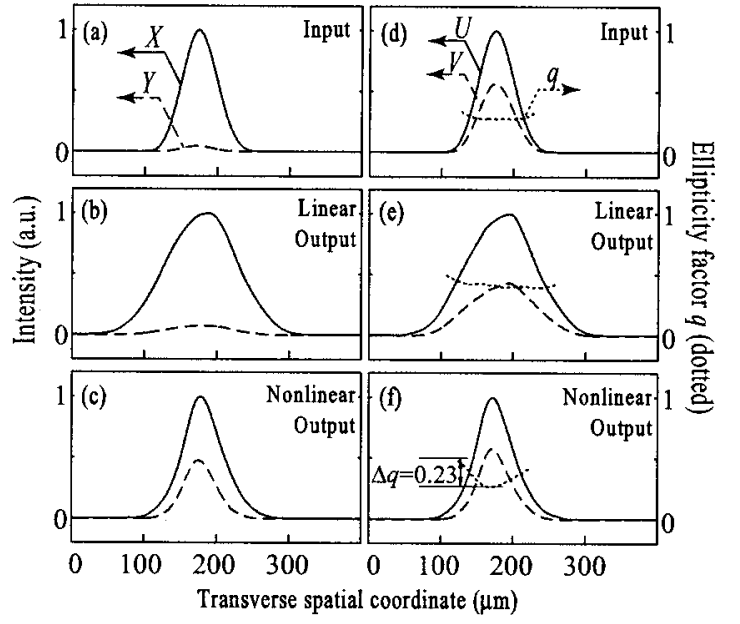

Fig. 2. Experimental results. (a), (b), (c) $E_{x}$ (solid curves) and $E_{y}$ (dashed curves), intensity profiles of, respectively, the input beam (FWHM, $49 \mu \mathrm{m}$ ), the output beam at low power (FWHM, $100 \mu \mathrm{m}$ ), and the output beam in the soliton regime (FWHM, $51 \mu \mathrm{m}$ ). (d), (e), (f) Intensity profiles for left-handed $U$ (solid curves) and right-handed $V$ (dashed curves) circular polarization and ellipticity factor $q$ (dotted curve). Note that in the soliton regime $\operatorname{FWHM}(U)$ $=53 \mu \mathrm{m}$ is different from $\operatorname{FWHM}(V)=45 \mu \mathrm{m}$.

files after linear propagation within the waveguide. We note clear diffraction of the beam whose full width at half-maximum (FWHM) is twofold compared with that of the input beam. The elliptic polarization is still flat across the beam, and no polarization rotation occurs. Note, however, that a small change in the $U / V$ ratio appears because of the residual birefringence of optical elements in the setup.

When the soliton regime is reached, i.e., for a mean pump power of $4 \mathrm{~mW}$ (maximum intensity of 4.4 $\times 10^{11} \mathrm{~W} \mathrm{~m}^{-2}$ ), self-focusing compensates exactly for diffraction: the beam's output FWHM is equal to the input FWHM. The results of this soliton regime are presented in Figs. 2(c) and 2(f), which show clear reshaping of the input Gaussian beams into hyperbolic secant-shaped profiles at the output for both linear and circular polarization. In addition, the $E_{y}$ polarization shown in Fig. 2(c) has much more energy than in the linear regime, meaning global polarization rotation of the soliton. As the ellipticity factor is rather small, one can approximate global polarization orientation angle $\theta$ by $\tan ^{2}(\theta) \approx I x / I y$. Thus, in our experiment, the global soliton polarization rotation is $\theta_{\text {out }}$ $-\theta_{\text {in }} \approx \pi / 5$. Moreover, we can see from Fig. 2(f) that the polarization state is no longer uniform across the entire beam, and the ellipticity factor exhibits a difference of $\Delta q=0.23$ between the center and the wings of the beam, as predicted by theory. ${ }^{9}$ We also measured different widths for the $U$ and $V$ beams of 53 and $45 \mu \mathrm{m}$, respectively. As a matter of fact, nonlinear reshaping of the $U$ and $V$ circular polarizations of the beam occurs during propagation without energy exchange, leading to different widths for the $U$ and $V$ beams. At this point it is important to claim that the vector soliton is fully formed and stabilized. Note also that a complete experimental analysis of the polar- ization state of the EPVS by Stokes parameters is currently being conducted.

We have been able to reproduce these observations numerically with a set of two coupled NLS equations that govern propagation of polarized light in isotropic Kerr media. Let us rewrite the incoherently coupled NLS equations on the basis of circular polarization $^{9,10}$ :

$$
\begin{aligned}
& \frac{\partial U}{\partial z}=\frac{i}{2 k} \frac{\partial^{2} U}{\partial x^{2}}+i \gamma\left[(1-B)|U|^{2}+(1+B)|V|^{2}\right] U, \\
& \frac{\partial V}{\partial z}=\frac{i}{2 k} \frac{\partial^{2} V}{\partial x^{2}}+i \gamma\left[(1-B)|V|^{2}+(1+B)|U|^{2}\right] V .
\end{aligned}
$$

Here $z$ is the propagation direction coordinate and $x$ is the free transverse dimension of the planar waveguide. $U, V=\left(E_{x} \pm i E_{y}\right) / \sqrt{2}$ are the left-handed and the right-handed circularly polarized components of the electric field and $E_{x}$ and $E_{y}$ are the associated orthogonal linearly polarized components, respectively. $k$ is the effective wave vector, $\gamma=2 \pi n_{2} / \lambda_{0}$ is a nonlinear coefficient, $n_{2}=3.5 \times 10^{-18} \mathrm{~m}^{2} \mathrm{~W}^{-1}$ (see, e.g., Ref. 11 ) is the nonlinear refractive index, and $\lambda_{0}$ is the vacuum field wavelength. $B=\chi_{x y y x} / \chi_{x x x x}$ is the polarization susceptibility ratio. We consider here, for the Kerr nonlinearity, the molecular reorientation effect of $\mathrm{CS}_{2}$, which prevails in the picosecond regime. In this case $B=3 / 4$, and we can expect a strong nonlinear birefringence. ${ }^{12}$

We performed numerical simulations by using the above experimental parameters. The theoretical results are illustrated in Fig. 3 and show excellent agreement with the experimental results depicted in

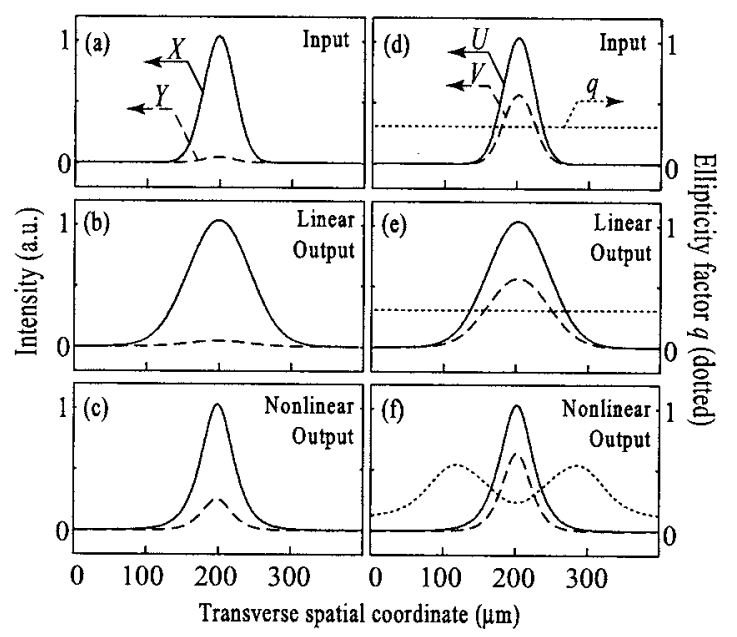

Fig. 3. Theoretical results. (a), (b), (c) $E_{x}$ (solid curves) and $E_{y}$ (dashed curves), intensity profiles for, respectively, the input beam (FWHM, $49 \mu \mathrm{m}$ ), the output beam at low power (FWHM, $100 \mu \mathrm{m}$ ), and the output beam in the soliton regime (FWHM, $49 \mu \mathrm{m}$ ). (d), (e), (f) Corresponding $U$ (solid curves), $V$ (dashed curves), and $q$ (dotted curves) profiles. Note that in the soliton regime $\operatorname{FWHM}(U)=53 \mu \mathrm{m}$ is different from $\operatorname{FWHM}(V)=49 \mu \mathrm{m}$. Parameters are $k=1.94$ $\times 10^{7} \mathrm{~m}^{-1}, \gamma=4.1 \times 10^{-11} \mathrm{~m} \mathrm{~W}^{-1}$, and maximum input intensity $I=4.5 \times 10^{11} \mathrm{~W} \mathrm{~m}^{-2}$. 


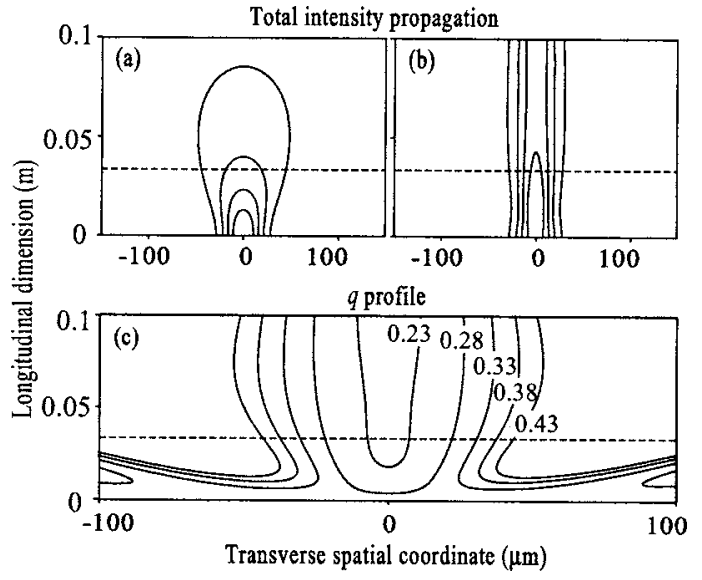

Fig. 4. Contour plots showing the evolution of (a) the diffracting beam, (b) the vector soliton, and (c) ellipticity factor $q$ in the soliton regime. The dashed lines correspond to the experiment and simulation of Figs. 2 and 3 , respectively.

Fig. 2. In particular, we can clearly see that there is no energy exchange between opposite circular polarization components in Figs. 2(e) and 2(f). We also retrieved theoretically the curvature of the ellipticity factor across the transverse profile $[\Delta q=0.3$; dotted curve in Fig. 2(f)], which is a specific feature of EPVSs propagating in a nonbirefringent medium because it results from mutual reshaping between opposite circular polarization.

To clearly identify soliton propagation, we numerically calculated in Fig. 4 the longitudinal evolution of the total field intensity (contour plots) in both the linear and the soliton regimes over a longer distance (10 $\mathrm{cm})$ than in Figs. 2 and $3(3 \mathrm{~cm})$. Figure 4(c) also shows a contour plot that illustrates the evolution of polarization ellipticity factor $q$ in the soliton regime. Once again the linear regime shows strong diffraction-induced beam widening, whereas the nonlinear regime exhibits soliton reshaping and further invariant propagation of the vector soliton. Moreover,
Fig. 4(c) shows that, once polarization ellipticity $q$ has decreased at the center of the spatial soliton, it remains constant all along the propagation.

To conclude, with the help of an original polarization-measurement setup we have confirmed experimentally the existence of the so-called elliptically polarized fundamental vector soliton theoretically predicted in 1994 for isotropic Kerr media. ${ }^{9}$ Our observations confirmed its characteristic polarization evolution and, in particular, the curve-shaped elliptic polarization profile across the beam. Finally, with a view to potential applications, one can deduce from these experiments that the nonlinear isotropic medium acts as a power-dependent variable and tunable wave plate. More fundamentally, the EPVS is the stable solution after symmetry breaking of multimode vector solitons in Kerr media, as described in Refs. 10 and 12 .

\section{References}

1. P. Maker, R. Terhune, and C. Savage, Phys. Rev. Lett. 12, 507 (1964).

2. H. Winful, Opt. Lett. 11, 33 (1986).

3. G. Gregori and S. Wabnitz, Phys. Rev. Lett. 56, 600 (1986).

4. B. Malomed, Phys. Rev. A 43, 410 (1991).

5. N. Akhmediev, A. Buryak, and J. Soto-Crespo, Opt. Commun. 112, 278 (1994).

6. Y. Barad and Y. Silberberg, Phys. Rev. Lett. 78, 3290 (1997).

7. D. Christodoulides and R. Joseph, Opt. Lett. 13, 53 (1988).

8. M. Tratnik and J. Sipe, Phys. Rev. A 38, 2011 (1988).

9. M. Haelterman and A. Sheppard, Phys. Lett. A 194, 191 (1994).

10. C. Cambournac, T. Sylvestre, H. Maillotte, B. Vanderlinden, P. Kockaert, P. Emplit, and M. Haelterman, Phys. Rev. Lett. 89, 083901 (2002).

11. C. Cambournac, H. Maillotte, E. Lantz, J. Dudley, and M. Chauvet, J. Opt. Soc. Am. B 19, 574 (2002).

12. M. Delqué, M. Chauvet, H. Maillotte, and T. Sylvestre, Opt. Commun. 249, 285 (2005). 